\title{
Valoración de las propiedades magnéticas en bioindicadores y en polvo urbano, como método alternativo para monitorear la contaminación atmosférica en zonas de flujo vehicular: Resultados preliminares sobre metales pesados en bioindicadores
}

\section{Assessment of magnetic properties in bioindicators and in urban dust, as an alternative method to monitor atmospheric pollution in areas of vehicular flow: Preliminary results about heavy metal in bioindicators}

Teresa Salazar-Rojas ${ }^{1}$, Guillermo Calvo-Brenes²

Salazar-Rojas, T; Calvo-Brenes, G. Valoración de las propiedades magnéticas en bioindicadores y en polvo urbano, como método alternativo para monitorear la contaminación atmosférica en zonas de flujo vehicular:

Resultados preliminares sobre metales pesados en bioindicadores. Tecnología en Marcha. Vol. 32, Especial. VIII

Encuentro de Investigación y Extensión. Abril 2019.

Pág 55-64.

DOI: https://doi.org/10.18845/tm.v32i6.4229

1 Centro de Investigación en Protección Ambiental (CIPA), Escuela de Química, Instituto Tecnológico de Costa Rica. Costa Rica. Estudiante doctorado DOCINADE. Correo electrónico: tsalazar@tec.ac.cr

2 Centro de Investigación en Protección Ambiental (CIPA), Escuela de Química, Instituto Tecnológico de Costa Rica. Costa Rica. Correo electrónico: gcalvo@tec.ac.cr 


\title{
Palabras clave
}

Contaminación del aire; metales pesados; bioindicadores.

\section{Resumen}

Tres de las mayores tendencias económicas de las últimas décadas han sido el crecimiento poblacional, industrial y urbanístico. Esta tendencia a provocado el incremento de emisiones de gases que contaminan el aire, afectando la salud humana. Las emisiones contaminan el aire con partículas en suspensión (PM) así como metales pesados, los cuales son retenidos en estas partículas. El monitoreo de la contaminación en el aire por métodos tradicionales es costoso; por otra parte, el uso de bioindicadores para medir la contaminación en el aire ha sido reportado. Los bioindicadores seleccionados fueron el ciprés, el limón agrio y el laurel de la India. El objetivo del proyecto es generar un método alternativo novedoso, de bajo costo y de fácil aplicación para monitorear la contaminación atmosférica en zonas con flujo vehicular. Se determinó el contenido de metales pesados en los 3 bioindicadores seleccionados, en distintas zonas del Gran Área Metropolitana (GAM). También se evaluaron las posibles correlaciones que existan entre cada una de estos metales con la densidad vehicular en los puntos de muestreo. Los resultados preliminares presentaron una correlación entre el $\mathrm{Ni}$ contenido en hojas de Ciprés con respecto a la Densidad Vehicular evaluado estadísticamente con un nivel de confianza del 95\%.

\section{Keywords}

Air pollution; heavy metals; bioindicators.

\begin{abstract}
Three of the greatest economic trends of the last decades have been the population, industrial and urban growth. This tendency has caused the increase of gas emissions that pollute the air, affecting human health. The emissions pollute the air with suspended particles (PM) as well as heavy metals, which are retained in these particles. Monitoring air pollution by traditional methods is expensive; on the other hand, the use of bioindicators to measure pollution in air has been reported. The bioindicators selected were cypress, bitter lemon and laurel from India. The objective of the project is to generate a novel alternative method, low in cost and easy application to monitor air pollution in areas with vehicular flow. The content of heavy metals in various bioindicators, was determined in different areas of the Greater Metropolitan Area (GAM). Analysis of possible correlations that may exist between each metal vehicle density at sampling points was evaluated. The preliminary results presented a correlation between the niquel (Ni) content in cypress leaves with respect to the Vehicle Density evaluated statistically with a confidence level of $95 \%$.
\end{abstract}

\section{Introducción}

El crecimiento poblacional, industrial y urbanístico ha llevado a un crecimiento en la flotilla vehicular, que es responsable por el 22-25\% de las emisiones de gases de efecto invernadero y un decrecimiento en la salud pública, por contribuir a enfermedades respiratorias y la diversificación de contaminantes ambientales [1] [2]. Se estima que unas 1500 millones de personas viven en áreas con niveles peligrosamente elevados de contaminación del aire [3] y que esa exposición de las personas produce la muerte de 7 millones de personas por año en el mundo [2]. 
Las partículas derivadas de las emisiones de gases vehiculares contienen elementos potencialmente tóxicos y material magnético. Existen dos tipos de partículas en suspensión: las menores a 10 micras $\left(\mathrm{PM}_{10}\right)$ y las menores a 2.5 micras $\left(\mathrm{PM}_{2.5}\right)$; siendo estas últimas potencialmente peligrosas, ya que pueden ingresar al sistema respiratorio, depositándose en tráquea y pulmones, e incluso pueden llegar al torrente sanguíneo y generar diversos problemas de salud [4] [2]. La emisión de partículas atmosféricas también está asociado a algunas actividades industriales. La combustión de combustibles fósiles da como resultado la dispersión de un amplio número de metales pesados, como son plomo $(\mathrm{Pb})$, cadmio $(\mathrm{Cd})$, cromo $(\mathrm{Cr})$, zinc (Zn), arsénico (As), antimonio (Sb), selenio (Se), bario (Ba), cobre (Cu), manganeso (Mn), uranio $(U)$ y vanadio $(V)$, sobre un gran área [5]. También, el uso de fertilizantes y plaguicidas contribuyen a la contaminación de metales que se encuentran en el suelo y son arrastrados

a la atmósfera por el viento, donde pueden ser retenidos en el material particulado. El $\mathrm{PM}_{2.5}$ puede ser absorbido por los humanos, mientras que el $\mathrm{PM}_{10}$ puede caer a tierra y contaminar las plantas y el suelo.

El uso de plantas como bioindicadores para la identificación de la contaminación en los diferentes medios, agua, suelo y aire ha sido reportado en muchos estudios científicos. El biomonitoreo permite establecer el impacto de la contaminación sobre los seres vivos en el ambiente, a diferencia de los métodos tradicionales que solo evalúan su parte abiótica (aire, agua, suelo). En cambio, el monitoreo convencional del ambiente urbano e industrial requiere de técnicas cada vez más costosas y complejas, por lo cual se hace necesario el uso de mecanismos alternativos que permitan obtener información relevante a precios más accesibles [6], [7], [8], [9].

De acuerdo con Thurman (1981) mencionado por [10] existen algunas especies que son capaces de ligar los metales a las paredes celulares o introducirlos en las vacuolas o acomplejarlos con ácidos orgánicos o sufren adaptaciones enzimáticas que les permiten realizar sus funciones en presencia de cantidades elevadas de metales pesados. Así dentro de la clasificación de las plantas existen las del tipo indicadoras que pueden reflejar el incremento de metal producido en el entorno. Asimismo, existen las acumuladoras que incrementan activamente metales en sus tejidos [10].

El empleo de bioindicadores ha resultado eficaz como muestreadores pasivos para evaluar la calidad del aire, convirtiéndose también en un método de bajo costo [11]. El objetivo del proyecto es generar un método alternativo novedoso, de bajo costo, de fácil aplicación y menos contaminante al ambiente, para monitorear la contaminación atmosférica en zonas de flujo vehicular.

\section{Metodología}

La metodología descrita en este artículo se enfoca en uno de los objetivos del proyecto que es la evaluación del uso de 3 bioindicadores ubicados en 5 distintos puntos de muestreo seleccionados. Los muestreos se llevarán a cabo en dos períodos de un año cada uno con muestreos bimensuales. En cada período se evaluará la pertinencia del uso de cada bioindicador seleccionado.Se presentan algunos resultados iniciales obtenidos en el análisis de contenido de metales pesados en los 3 bioindicadores. La metodología que se menciona a continuación está relacionada con estos análisis preliminares. 


\section{Selección de puntos de muestreo}

El estudio se llevó a cabo dentro del GAM donde vive el 50\% de la población del país considerando la densidad vehicular (número de vehículos/día). También se hizo un inventario de los distintos tipos de arbustos y árboles en cada zona con el apoyo de un experto en plantas del Instituto Tecnológico de Costa Rica.

Se seleccionaron 5 puntos de muestreo considerando que tuvieran diferente densidad vehicular y que estuvieran representados los bioindicadores seleccionados. Estos puntos fueron: La Universidad Técnica Nacional (UTN) en Alajuela, la Riviera de Belén en Heredia, Ochomogo por Recope, Cartago, Hatillo en San José y Coronado en San José.

\section{Bioindicadores}

Se seleccionaron 3 especies de plantas como bioindicadores, considerando criterios de abundancia, accesibilidad, longevidad, características morfológicas de las hojas que se consideren óptimas para la captura y retención de contaminantes. Para esta labor de reconocimiento de las plantas, se contó con la colaboración de un experto. Los bioindicadores seleccionados fueron: el ciprés (Cupressus lusitanica), el limón agrio (género Citrus) y el laurel de la India (Ficus benjamina L.). La selección de estos bioindicadores se basó en estudios de fuentes bibliográficas y estudios científicos [7], [12] que demuestran que ciertas plantas tienen la capacidad de absorber estos metales pesados. Otro criterio empleado fue que cada bioindicador seleccionado debió estar presente en cada uno de los 5 sitios de muestreo.

\section{Metales pesados}

Los metales pesados seleccionados para el análisis de su contenido en bioindicadores fueron el: $\mathrm{Cr}, \mathrm{Cu}, \mathrm{Ni}, \mathrm{Pb}, \mathrm{V}, \mathrm{Zn}$ y Fe que son metales contaminantes usualmente presentes en el aire. La metodología de análisis del contenido de metales pesados fue el de la EPA Method 3050b Acid Digestion Of Sediments, Sludges, And Soils [13]. Las mediciones de contenido de metales pesados fueron llevadas a cabo en un espectrofotómetro de Absorción Atómica Shimatzu, con el apoyo de un horno de grafito y un generador de hidruros, para la determinación de concentraciones a nivel de trazas.

\section{Análisis estadístico}

El análisis de posibles correlaciones entre la Densidad Vehicular con respecto a cada uno de los metales se llevó a cabo utilizando el Coeficiente de Correlación de Pearson con un nivel de confianza del 95\% $(a=0,05)$. El programa de cómputo empleado para tal fin fue el Statistical Package for Social Science (SPSS), versión 25.

\section{Resultados y discusión}

El cuadro 1 muestra resultados del análisis de contenido de metales pesados en 3 bioindicadores, ubicados en cada uno de los 5 puntos de muestreo. La densidad vehicular en las zonas de estudio corresponde a valores que van desde los 2180 hasta los 60591 vehículos promedio por día. La concentración de cada metal analizado está expresada en miligramos de metal por gramo de bioindicador (en base seca).

Las concentraciones de $\mathrm{Pb}$ en el material vegetal muestra valores bajos; igual situación muestra el $\mathrm{Cr}$ y el $\mathrm{Ni}$, mientras que el Fe fue el metal con las mayores concentraciones. 
Cuadro 1. Concentración de metales pesados en 3 distintos bioindicadores.

\begin{tabular}{|c|c|c|c|c|c|c|c|c|c|}
\hline \multirow{2}{*}{ Bioindicador } & \multirow{2}{*}{ Ubicación } & \multirow{2}{*}{$\begin{array}{c}\text { Densidad } \\
\text { vehicular } \\
\text { (\#/día) }\end{array}$} & \multicolumn{7}{|c|}{ Concentración del metal $(\mathrm{mg} / \mathrm{g})$} \\
\hline & & & $\mathrm{Fe}$ & $\mathrm{Cu}$ & $\mathrm{Pb}$ & $\mathrm{Cr}$ & $\mathrm{Ni}$ & V & $\mathrm{Zn}$ \\
\hline \multirow{5}{*}{ Ciprés } & Coronado & 2180 & 0.0948 & 0.0330 & 0.0000 & 0.0009 & 0.0008 & 0.0012 & 0.0441 \\
\hline & Alajuela & 14993 & 0.1950 & 0.0000 & 0.0000 & 0.0009 & 0.0012 & 0.0041 & 0.0203 \\
\hline & Belén & 21182 & 0.2834 & 0.0333 & 0.0004 & 0.0017 & 0.0012 & 0.0057 & 0.0267 \\
\hline & Ochomogo & 43512 & 1.2685 & 0.0000 & 0.0057 & 0.0042 & 0.0012 & 0.0041 & 0.0496 \\
\hline & Hatillo & 60591 & 0.0863 & 0.0247 & 0.0000 & 0.0007 & 0.0019 & 0.0024 & 0.0283 \\
\hline \multirow{5}{*}{ Cítrico } & Coronado & 2180 & 0.1073 & 0.0330 & 0.0000 & 0.0006 & 0.0013 & 0.0044 & 0.0359 \\
\hline & Alajuela & 14993 & 0.1903 & 0.0207 & 0.0017 & 0.0020 & 0.0000 & 0.0031 & 0.0261 \\
\hline & Belén & 21182 & 0.0946 & 0.0411 & 0.0000 & 0.0005 & 0.0002 & 0.0034 & 0.0378 \\
\hline & Ochomogo & 43512 & 0.2231 & 0.0000 & 0.0025 & 0.0012 & 0.0023 & 0.0026 & 0.0306 \\
\hline & Hatillo & 60591 & 0.1597 & 0.0246 & 0.0000 & 0.0011 & 0.0009 & 0.0032 & 0.0184 \\
\hline \multirow{5}{*}{ Ficus } & Coronado & 2180 & 0.2923 & 0.0329 & 0.0006 & 0.0009 & 0.0008 & 0.0038 & 0.0263 \\
\hline & Alajuela & 14993 & 0.1245 & 0.0166 & 0.0017 & 0.0009 & 0.0010 & 0.0043 & 0.0174 \\
\hline & Belén & 21182 & 0.1213 & 0.0335 & 0.0000 & 0.0007 & 0.0003 & 0.0029 & 0.0176 \\
\hline & Ochomogo & 43512 & 0.2089 & 0.0000 & 0.0037 & 0.0009 & 0.0000 & 0.0056 & 0.0292 \\
\hline & Hatillo & 60591 & 0.1075 & 0.0331 & 0.0004 & 0.0006 & 0.0012 & 0.0031 & 0.0252 \\
\hline
\end{tabular}

Las figuras 1 al 7 muestran el comportamiento del contenido de los metales analizados, según el tipo de bioindicador y por sitio de muestreo. Se conoce que la retención de metales en las hojas depende del tipo de bioindicador; sin embargo, no se observa un comportamiento específico entre los distintos bioindicadores que nos indique que un bioindicador retiene un determinado metal mejor que los otros.

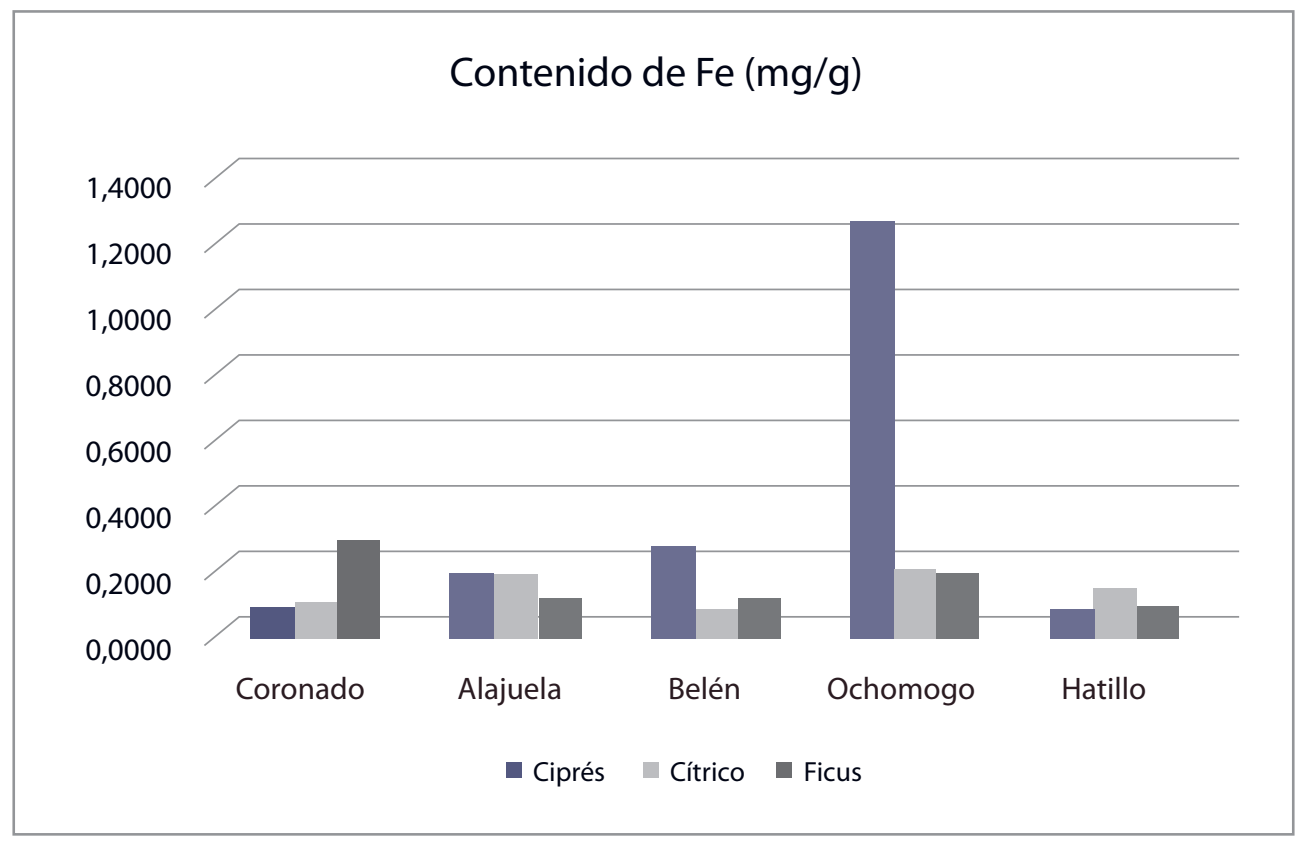

Figura 1. Contenido de Fe por bioindicador según el sitio de muestreo 


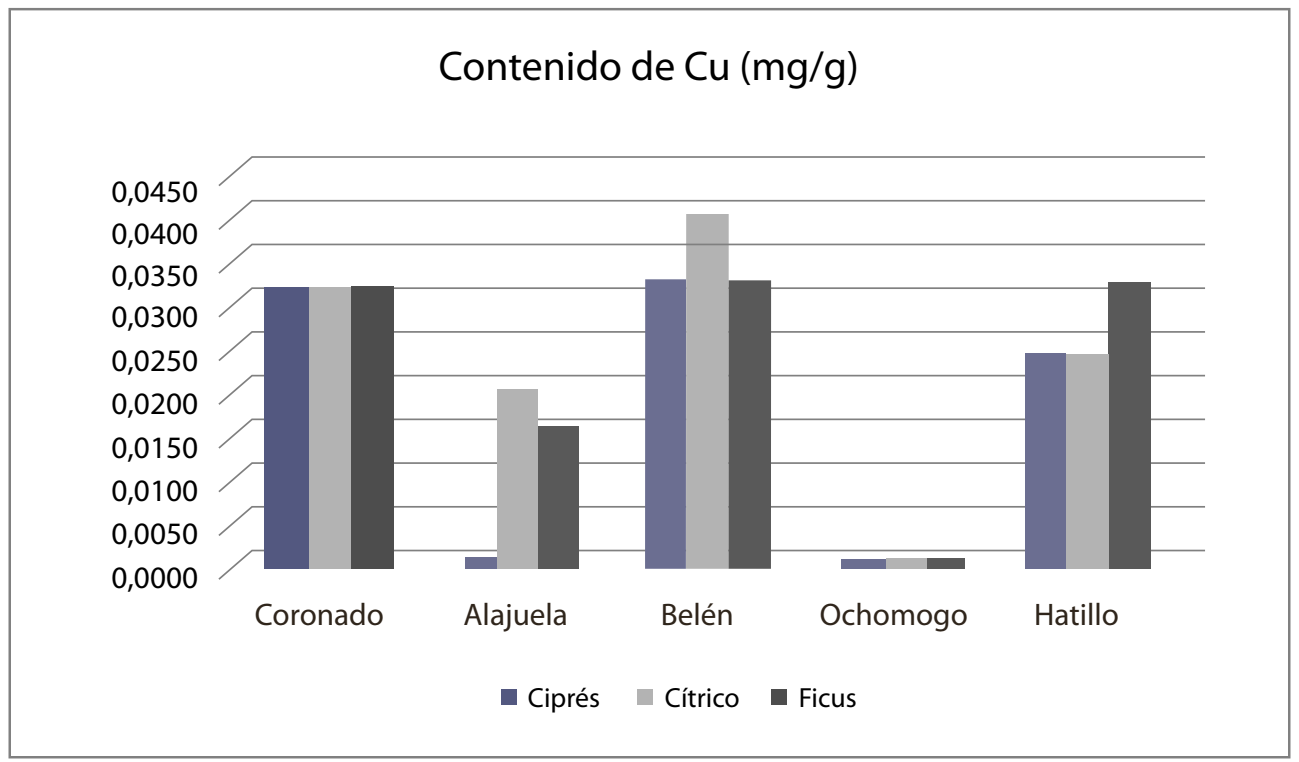

Figura 2. Contenido de Cu por bioindicador según el sitio de muestreo

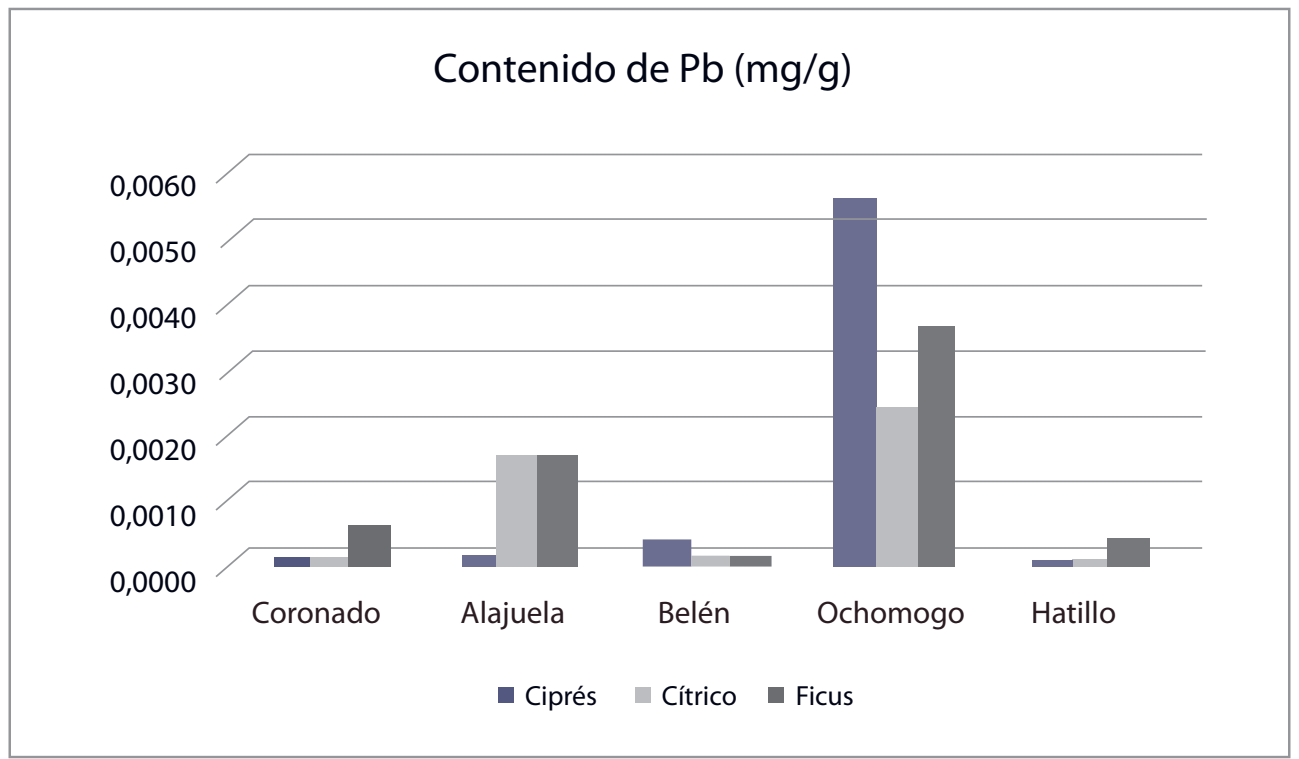

Figura 3. Contenido de $\mathrm{Pb}$ por bioindicador según el sitio de muestreo 


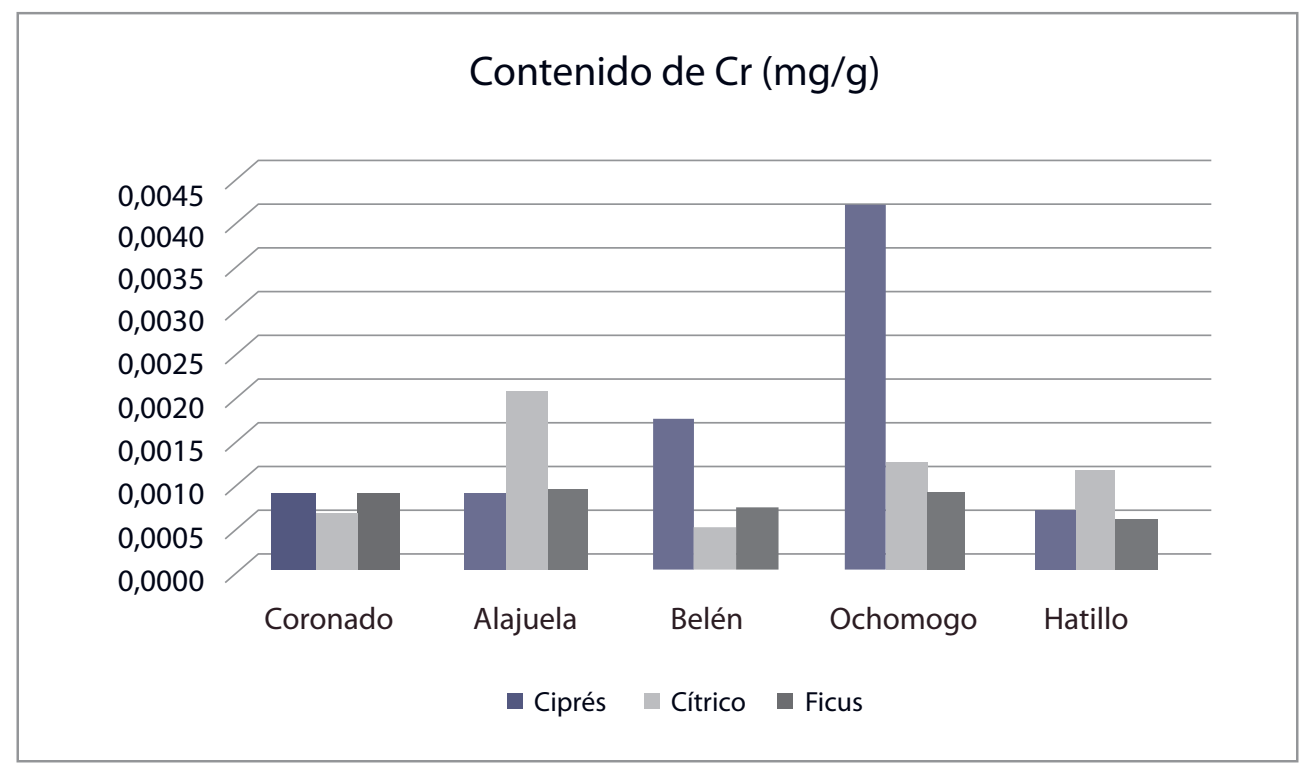

Figura 4. Contenido de Cr por bioindicador según el sitio de muestreo

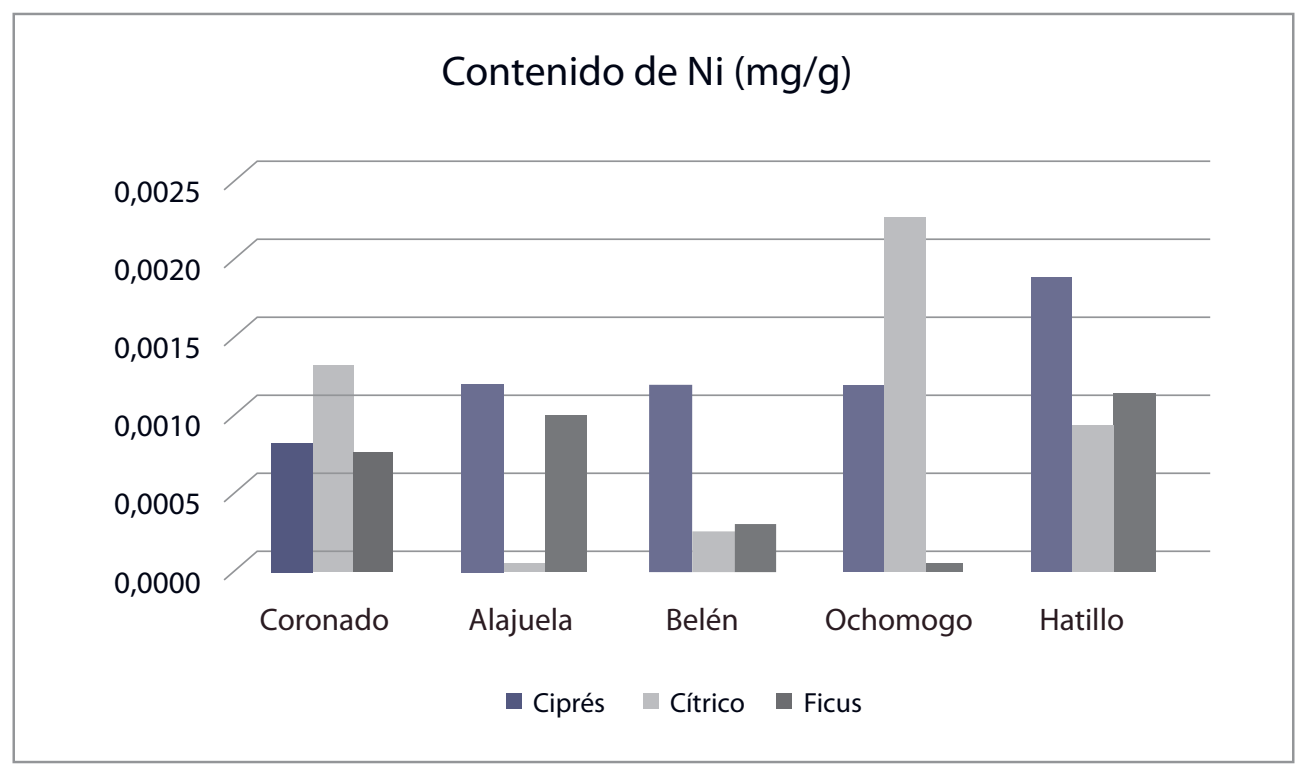

Figura 5. Contenido de Ni por bioindicador según el sitio de muestreo 


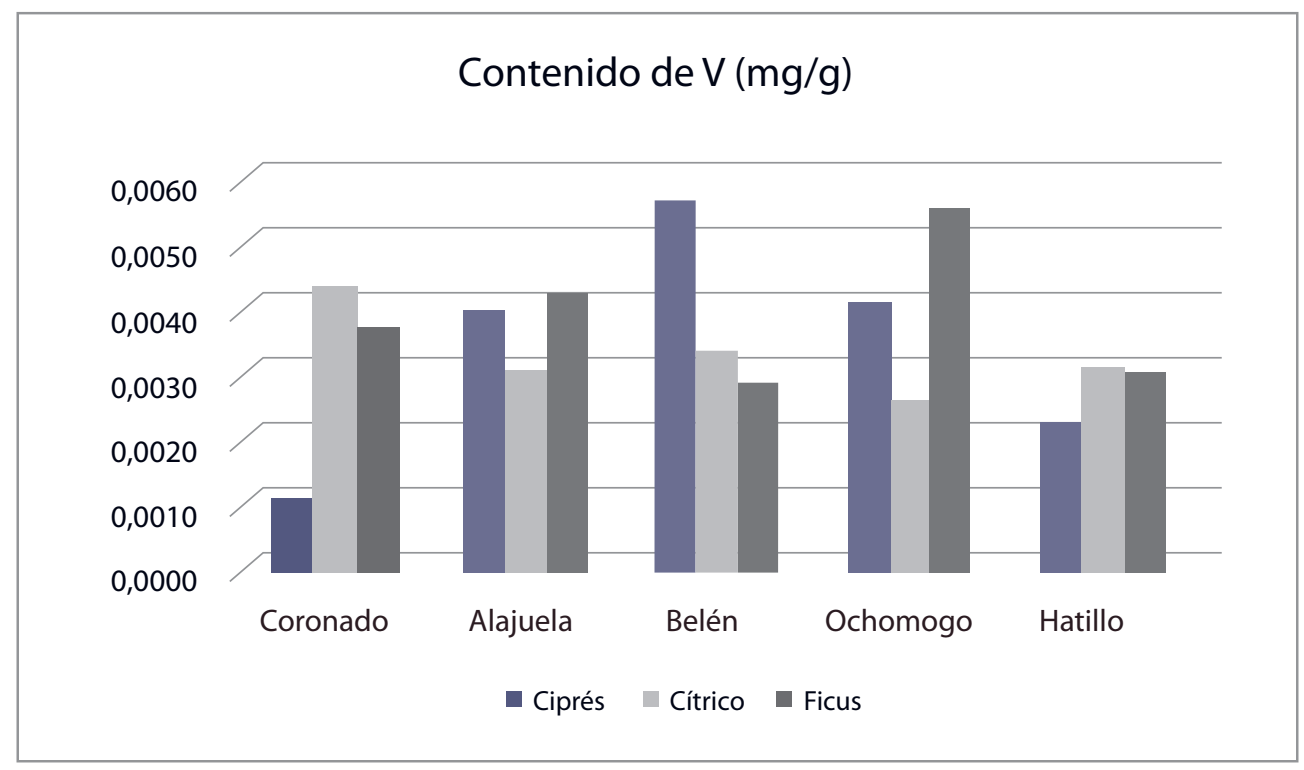

Figura 6. Contenido de V por bioindicador según el sitio de muestreo

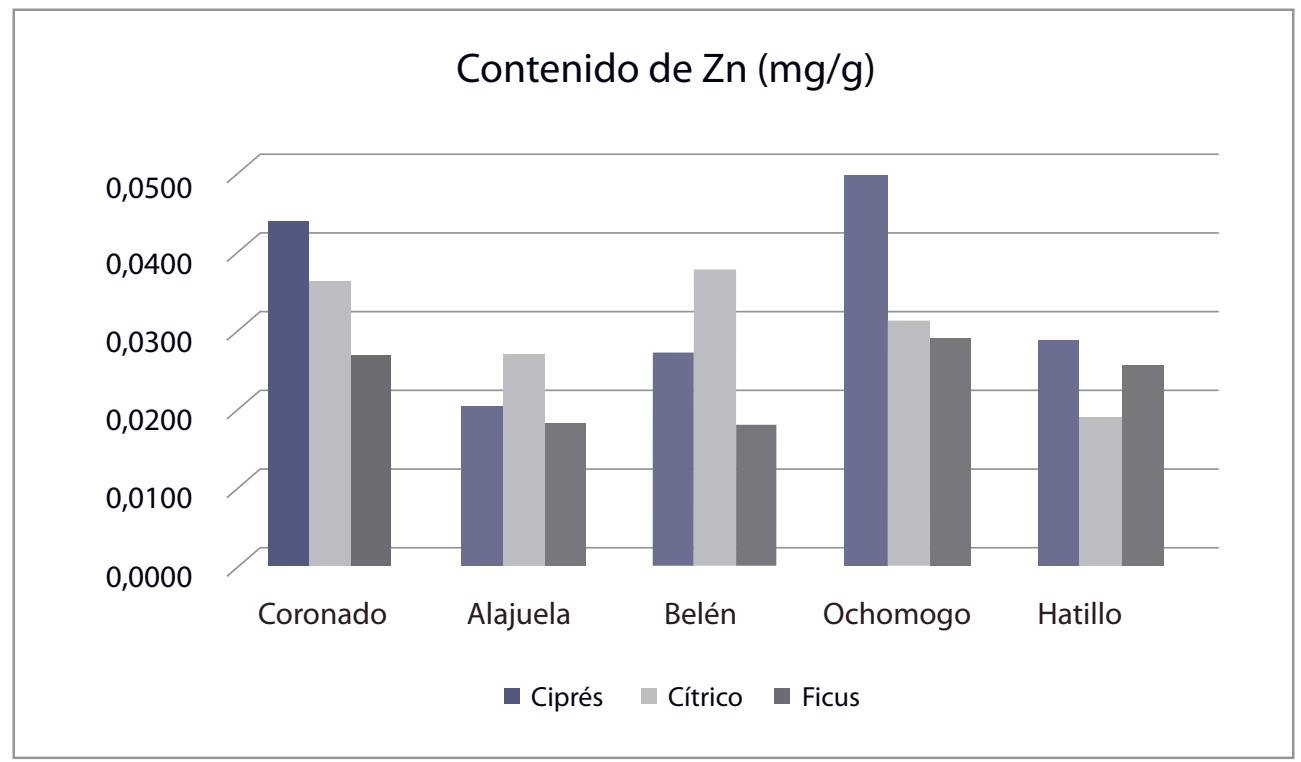

Figura 7. Contenido de Zn por bioindicador según el sitio de muestreo

Los sitios de muestreo en los gráficos están ordenados en forma ascendente con respecto a la densidad vehicular: Coronado con una densidad de 2180 vehículos/día, le sigue Alajuela con 14993, Belén con 21182, Ochomogo con 43512 y finalmente Hatillo con 60591. En general, parece ser que no hay una tendencia entre el contenido de cada uno de los metales analizados en los bioindicadores con respecto a la densidad vehicular.

El análisis estadístico de Densidad Vehicular con cada uno de los metales evaluados en el Ciprés, indica que no hay correlación entre la Densidad Vehicular con respecto al Fe, Cu, Pb, Cr, V y Zn $(a=0,05)$ cuando el bioindicador fue el Ciprés. En el caso del Ni este si mostró una correlación positiva con la Densidad Vehicular (figura 8) y el nivel de confianza fue de 95,6\%. 


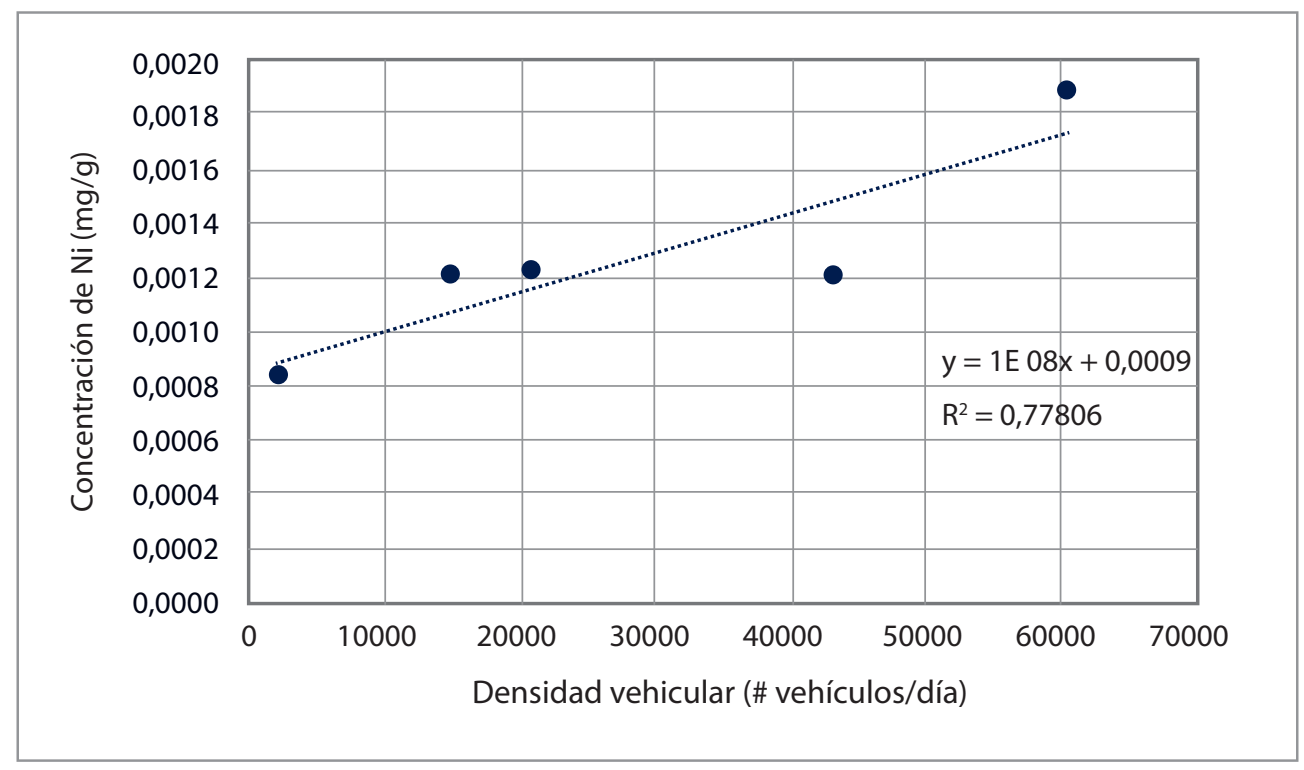

Figura 8. Comportamiento de la concentración de Ni en Ciprés con relación a la densidad vehicular

En el caso de los metales evaluados en las hojas de Cítricos y Ficus el análisis estadístico mostró que ninguno de los metales tenía una correlación significativa con relación a la densidad vehicular $(\mathrm{a}=0,05)$.

\section{Conclusiones}

De los metales evaluados, el Ni retenido en el Ciprés fue el único que mostró correlación entre su concentración y la Densidad Vehicular. Este comportamiento está sujeto al tipo de bioindicador seleccionado por lo que no se puede descartar que el uso de otros bioindicadores no evaluados en esta investigación puedan mostrar una correlación mejor con relación a esta variable.

\section{Recomendaciones}

Se recomienda evaluar otros bioindicadores que podrían mostrar una tendencia con respecto a la Densidad Vehicular.

Se debe determinar la relación existente entre contenido de metales pesados en aire, polvo urbano, y contenido de PM con respecto a la Densidad Vehicular para poder determinar si existen correlaciones entre cada uno de ellos para conocer mejor la dinámica que muestran entre sí. Se deben evaluar las propiedades magnéticas en bioindicadores y polvo urbano como un método alternativo en el monitoreo de contaminantes en el aire y su relación con las otras variables estudiadas. Estos aspectos forman parte del proyecto y serán evaluados posteriormente.

Se debe verificar si la intensidad de la lluvia en el período seco y el lluvioso tiene alguna influencia en los resultados. Las muestras analizadas correspondieron a la época de mayor intensidad de lluvia por lo que es importante evaluar los resultados provenientes de la época seca. 


\section{Agradecimientos}

Los autores expresan su agradecimiento al Instituto Tecnológico de Costa Rica (ITCR). También, al Centro de Investigación en Protección Ambiental (CIPA) y al Laboratorio de Servicios Químicos y Microbiológicos (CEQIATEC) por su gran respaldo a esta investigación. Agradecemos al Master Luis Guillermo Acosta Vargas, de la Escuela de Ingeniería Forestal, por su apoyo en el levantamiento de inventario de plantas durante esta etapa de la investigación. También se agradece a la Refinadora Costarricense de Petróleo (RECOPE), por la cooperación brindada, en los muestreos realizados en la zona de Ochomogo.

\section{Referencias}

[1] M. Carley y I. Christie, Managing sustainable development. Routledge, 2017.

[2] S. Ramos. Los metales pesados: importancia de los estudios en áreas urbanas y periurbanas. Primer congreso internacional de geología médica. Tuxtla Gutiérrez, Chiapas. México. 2016. Recuperado Mayo 28, 2016 desde http://www.sgm.gob.mx/pdfs/26 10\%20Los\%20Metales\%20Pesados\%20Est areas\%20urbanas\%20 y\%20periurbanas.pdf

[3] C. Chelala, Impacto del ambiente sobre la salud infantil. OPS, 1999.

[4] A. Sánchez-Duque et al., «Evaluación de la contaminación ambiental a partir del aumento magnético en polvos urbanos. Caso de estudio en la ciudad de Mexicali, México», Rev. Mex. Cienc. Geológicas, vol. 32, n.o 3, pp. 501-513, 2015.

[5] A. M. Ure, «Heavy Metal in Soil, ed», BJ Allow. Blackie Sons Ltd, vol. 40, 1990.

[6] G. Wolff, G. C. Pereira, E. M. Castro, J. Louzada, y F. F. Coelho, «The use of Salvinia auriculata as a bioindicator in aquatic ecosystems: biomass and structure dependent on the cadmium concentration», Braz. J. Biol., vol. 72, n.o 1, pp. 71-77, 2012.

[7] C. S. Fontanetti, L. R. Nogarol, R. B. de Souza, D. G. Perez, y G. T. Maziviero, «Bioindicators and biomarkers in the assessment of soil toxicity», en Soil contamination, InTech, 2011.

[8] Z. O. Ojekunle, M. Adeboje, A. G. Taiwo, R. O. Sangowusi, A. M. Taiwo, y V. O. Ojekunle, «Tree Leaves as Bioindicator of Heavy Metal Pollution in Mechanic Village, Ogun State.», J. Appl. Sci. Environ. Manag., vol. 18, n.o 4, pp. 639-644, 2014.

[9] R. LijteRoff, L. Lima, y B. PRieRi, «Uso de líquenes como bioindicadores de contaminación atmosférica en la ciudad de San Luis, Argentina», Rev. Int. Contam. Ambient., vol. 25, n.o 2, pp. 111-120, 2009.

[10] M. Peris, «Estudio de metales pesados en suelos bajo cultivos hortícolas de la provincia de Castellón», Univ. Valencia Esp., 2006.

[11] B. Aguilar Reyes et al., «Reconnaissance environmental magnetic study of urban soils, dust and leaves from Bogotá, Colombia», Stud. Geophys. Geod., vol. 57, n.o 4, pp. 741-754, 2013.

[12] B. Aguilar Reyes et al., «Ficus benjamina leaves as indicator of atmospheric pollution: a reconaissance study», Stud. Geophys. Geod., vol. 56, n.o 3, pp. 879-887, 2012.

[13] U.S. Environmental Protection Agency. EPA Method 3050b Acid Digestion of Sediments, Sludges, and Soils. 2017. 